\title{
Evaluations of a Weighted Average of Gauss Sums
}

\author{
Wen-Kai Shao $\mathbb{D D}^{1}$ and Yuan He $\mathbb{D}^{2}$ \\ ${ }^{1}$ Department of Mathematical Teaching and Research, Yibin Vocational \& Technical College, Yibin 644003, Sichuan, China \\ ${ }^{2}$ School of Mathematics and Information Science, Neijiang Normal University, Neijiang 641100, Sichuan, China
}

Correspondence should be addressed to Yuan He; hyyhe@aliyun.com

Received 23 May 2021; Accepted 17 June 2021; Published 30 June 2021

Academic Editor: Yuan Yi

Copyright $\odot 2021$ Wen-Kai Shao and Yuan He. This is an open access article distributed under the Creative Commons Attribution License, which permits unrestricted use, distribution, and reproduction in any medium, provided the original work is properly cited.

In this paper, we perform a further investigation for a weighted average of Gauss sums. By making use of some properties of the cotangent function and the Bernoulli polynomials, we explicitly evaluate the weighted average of Gauss sums in terms of the special values of Dirichlet $L$-functions at positive integers.

\section{Introduction}

Let $q$ be a positive integer, and let $\chi$ be a Dirichlet character modulo $q$. The Gauss sum associated with $\chi$ is defined for an arbitrary integer $n$ (see, e.g., Section 8.5 in [1]).

$$
G(n, \chi)=\sum_{r=1}^{q} \chi(r) e^{(2 \pi i r n / q)},
$$

where $i$ is the imaginary unit. In particular, the case $n=1$ in (1) is usually denoted by

$$
\tau(\chi)=\sum_{r=1}^{q} \chi(r) e^{(2 \pi i r / q)} .
$$

It is well known that various properties and applications of $\tau(\chi)$ appear in many books and papers. For example, an important result of $\tau(\chi)$ is as follows (see, e.g., Theorem 8.15 in [1]):

$$
|\tau(\chi)|=\sqrt{q},
$$

if $\chi$ is a primitive Dirichlet character modulo $q$. We here mention [2-5] for further explorations of (3). Moreover, it is also demonstrated that some weighted averages of $\tau(\chi)$ have good distribution properties when $\chi$ is a nonprimitive Dirichlet character modulo $q$. For example, let $k$ be a positive integer and let $m$ be a positive real number, and $\mathrm{Yi}$ and Zhang [6] used the estimate for character sums and the method of trigonometric sums to study the mean value of $|\tau(\chi)|^{m}$ with the weight of the inversion of $|L(1, \chi)|^{2 k}$, where $L(s, \chi)$ denotes the Dirichlet $L$-functions defined for a complex number $s$ and a Dirichlet character $\chi$ modulo $q$ by the series as follows:

$$
L(s, \chi)=\sum_{n=1}^{\infty} \frac{\chi(n)}{n^{s}}, \quad(\Re(s)>1),
$$

and gave a sharper asymptotic formula. After that Liu and Zhang [7] used some properties of primitive Dirichlet character and some mean value formulas of Dirichlet $L$-functions to study the high-order mean value of products of $\tau(\chi)$ and the generalized Bernoulli numbers and obtained an interesting asymptotic formula. We also refer to [8] for a further investigation for the high-order mean value of products of $\tau(\chi)$ and the generalized Bernoulli numbers. More recently, Alkan [9] used a special evaluation method for Dirichlet $L$-functions to consider the following weighted average of Gauss sums:

$$
A_{\chi}(\omega)=\frac{1}{q} \sum_{r=1}^{q} \omega\left(\frac{r}{q}\right) G(r, \chi),
$$

where $\omega(\cdot)$ is a real-valued function defined on the interval $[0,1]$ and depicted that the weighted averages of Gauss sums $A_{\chi}(\omega)$ and $i A_{\chi}(\omega)$ and the character values $\chi(m)$ at positive integers $m$ can be well approximated by linear combinations 
of the algebraic parts of special values of Dirichlet $L$-functions under correct parity conditions (see, e.g., Theorems 1, 2, and 3 in [9]). The special evaluation method for Dirichlet $L$-functions posed by Alkan (see Theorem 4 in [9]) states that if $\omega(x)=x^{m}$ for a positive integer $m$, then for a nonprincipal even Dirichlet character $\chi$ modulo $q$,

$$
A_{\chi}(\omega)= \begin{cases}C_{2, m} \frac{L(2, \chi)}{\pi^{2}}+C_{4, m} \frac{L(4, \chi)}{\pi^{4}}+\cdots+C_{m, m} \frac{L(m, \chi)}{\pi^{m}}, & 2 \mid m, \\ C_{2, m}^{\times} \frac{L(2, \chi)}{\pi^{2}}+C_{4, m}^{\times} \frac{L(4, \chi)}{\pi^{4}}+\cdots+C_{m-1, m}^{\times} \frac{L(m-1, \chi)}{\pi^{m-1}}, & 2+m,\end{cases}
$$

and for an odd Dirichlet character $\chi$ modulo $q$,

$$
A_{\chi}(\omega)= \begin{cases}D_{1, m} \frac{L(1, \chi)}{\pi}+D_{3, m} \frac{L(3, \chi)}{\pi^{3}}+\cdots+D_{m, m} \frac{L(m, \chi)}{\pi^{m}}, & 2+m, \\ D_{1, m}^{\times} \frac{L(1, \chi)}{\pi}+D_{3, m}^{\times} \frac{L(3, \chi)}{\pi^{3}}+\cdots+D_{m-1, m}^{\times} \frac{L(m-1, \chi)}{\pi^{m-1}}, & 2 \mid m,\end{cases}
$$

where the coefficients $C_{2 k, m}, C_{2 k, m}^{\times} \in \mathbb{Q}$ for a positive integer $k$ with $2 k \leq m$ and the coefficients $D_{2 j+1, m}, D_{2 j+1, m}^{\times} \in i \mathbb{Q}$ for a nonnegative integer $j$ with $2 j+1 \leq m$ can be computed recursively by using the Bernoulli numbers.

Motivated and inspired by the work of Alkan [9], in this paper, we use some properties of the cotangent function and the Bernoulli polynomials to study the above weighted average of Gauss sums and determine the coefficients appearing in (6) and (7) which can be explicitly evaluated by the binomial coefficients. The precise statements of our results are as follows.

Theorem 1. Let $\omega(x)=x^{m}$ for a positive integer $m$. Then, for a nonprincipal even Dirichlet character $\chi$ modulo $q$,

$$
A_{\chi}(\omega)=-\frac{1}{m+1} \sum_{j=1}^{\lfloor m / 2\rfloor}\left(\begin{array}{c}
m+1 \\
2 j
\end{array}\right)(-1)^{j} \frac{(2 j) ! \cdot L(2 j, \chi)}{\pi^{2 j} \cdot 2^{2 j-1}},
$$

and for an odd Dirichlet character $\chi$ modulo $q$,

$$
A_{\chi}(\omega)=-\frac{i}{m+1} \sum_{j=0}^{\lfloor(m-1 / 2)\rfloor}\left(\begin{array}{c}
m+1 \\
2 j+1
\end{array}\right)(-1)^{j} \frac{(2 j+1) ! \cdot L(2 j+1, \chi)}{\pi^{2 j+1} \cdot 2^{2 j}},
$$

where $\lfloor x\rfloor$ denotes the greatest integer less than or equal to real number $x$, and the sum on the right hand side of (8) vanishes when $m=1$.

It becomes obvious that Theorem 1 gives the following explicit evaluations of the coefficients appearing in Alkan's [9] formulas (6) and (7).

Corollary 1. Let $k$ and $m$ be positive integers, and let $j$ be a nonnegative integer. Suppose that $C_{2 k, m}$ and $C_{2 k, m}^{\times}$are the same as those in (6) and $D_{2 j+1, m}, D_{2 j+1, m}^{\times}$are the same as those in (7). Then,

$$
\begin{aligned}
C_{2 k, m}=C_{2 k, m}^{\times} & =-\frac{(-1)^{k}}{m+1}\left(\begin{array}{c}
m+1 \\
2 k
\end{array}\right) \frac{(2 k) !}{2^{2 k-1}}, \\
D_{2 j+1, m} & =D_{2 j+1, m}^{\times}=-i \frac{(-1)^{j}}{m+1}\left(\begin{array}{c}
m+1 \\
2 j+1
\end{array}\right) \frac{(2 j+1) !}{2^{2 j}} .
\end{aligned}
$$

This paper is organized as follows. In the second section, we present some auxiliary results. The third section concentrates on the features that have contributions to the proof of Theorem 1.

\section{Some Auxiliary Results}

Before giving the proof of Theorem 1, we need the following auxiliary results.

Lemma 1. Let $q$ and $n$ be positive integers with $q \geq 2$, and let $\chi$ be a nonprincipal Dirichlet character modulo q. Then,

$$
\begin{aligned}
\sum_{r=1}^{q-1} \chi(r) \cot ^{n}\left(\frac{\pi r}{q}\right)= & \sum_{k=1}^{n} \frac{q^{k} A(n, k)}{\pi^{k}} L(k, \chi) \\
& +\chi(-1) \sum_{k=1}^{n}(-1)^{k} \frac{q^{k} A(n, k)}{\pi^{k}} L(k, \chi),
\end{aligned}
$$

where $A(n, k)$ is given for positive integers $n, k$ with $1 \leq k \leq n$ by

$$
A(n, k)=i^{n-k} \frac{(k-1) !}{2^{k}} \sum_{l=k}^{n}\left(\begin{array}{l}
n \\
l
\end{array}\right) \frac{2^{l} s(l, k)}{(l-1) !},
$$

with $s(l, k)$ being the Stirling numbers of the first kind.

Proof. (see Theorem 2.2 in [10] for details).

Remark 1. It is worth noticing that the finite trigonometric sum on the left hand side of (11) was also studied by Zhang and Lin [11], where a nice connection between the Dirichlet $L$-functions at even positive integers and the finite trigonometric sum on the left hand side of (11) is established, and some interesting identities involving finite trigonometric sums are deduced, and a new proof for the mean square value formula of Dirichlet L-functions showed in $[12,13]$ is also presented.

Lemma 2. Let $n$ be a positive integer, and let $\theta_{r}$ be a real function defined on a positive integer $r$. Then,

$$
\left.\frac{\partial^{n-1}}{\partial a^{n-1}}(\cot (\pi a))\right|_{a=\left(\theta_{r} / q\right)}=\frac{\delta_{1, n}}{i}+2^{n} \pi^{n-1} i^{n-2} F\left(\frac{\theta_{r}}{q}, 1-n\right),
$$

where $\delta_{1, n}$ is the Kronecker delta given by $\delta_{1, n}=1$ or 0 according to $n=1$ or $n \neq 1$, respectively, and $F(a, s)$ is the 
periodic zeta function given for a real number $a$ and $a$ complex number s by

$$
F(a, s)=\sum_{n=1}^{\infty} \frac{e^{2 \pi i n a}}{n^{s}}, \quad(\Re(s)>1) .
$$

Proof. (see Equation (2.28) in [10] for details).

Lemma 3. Let $q$ be a positive integer. Then, if $\chi$ is an even Dirichlet character modulo $q$, then for a positive integer $n$,

$$
L(2 n, \chi)=(-1)^{n-1} \frac{\pi^{2 n} \cdot 2^{2 n-1}}{q \cdot(2 n) !} \sum_{r=1}^{q} B_{2 n}\left(\frac{r}{q}\right) G(r, \chi)
$$

and if $\chi$ is an odd Dirichlet character modulo $q$, then for a nonnegative integer $n$,

$$
L(2 n+1, \chi)=(-1)^{n} i \frac{\pi^{2 n+1} \cdot 2^{2 n}}{q \cdot(2 n+1) !} \sum_{r=1}^{q} B_{2 n+1}\left(\frac{r}{q}\right) G(r, \chi),
$$

where $B_{n}(x)$ are the Bernoulli polynomials defined by the generating function (see, e.g., $[1,14]$ )

$$
\frac{t e^{x t}}{e^{t}-1}=\sum_{n=0}^{\infty} B_{n}(x) \frac{t^{n}}{n !}, \quad(|t|<2 \pi) .
$$

Proof. It is obvious from (4) to see that

$$
\begin{aligned}
L(s, \chi) & =\sum_{m=0}^{\infty} \sum_{l=1}^{q} \frac{\chi(q m+l)}{(q m+l)^{s}} \\
& =\frac{1}{q^{s}} \sum_{l=1}^{q} \chi(l) \zeta\left(s, \frac{l}{q}\right),
\end{aligned}
$$

where $\zeta(s, x)$ is the Hurwitz zeta function given with a real number $x>0$ and a complex number $s$ by

$$
\zeta(s, x)=\sum_{n=0}^{\infty} \frac{1}{(n+x)^{s}}, \quad(\Re(s)>1) .
$$

By taking $s=n$ in (18), we get that for a positive integer $n$,

$$
\begin{aligned}
& L(n, \chi)=\frac{1}{q^{n}} \sum_{l=1}^{q} \chi(l) \zeta\left(n, \frac{l}{q}\right) \\
& =\frac{\chi(-1)}{q^{n}} \sum_{l=1}^{q} \chi(l) \zeta\left(n, 1-\frac{l}{q}\right) .
\end{aligned}
$$

Since the Hurwitz zeta function at positive integers satisfies the reflection formula (see, e.g., Section 4 in [15] or equation (25) in [16]),

$$
\zeta(n, a)+(-1)^{n} \zeta(n, 1-a)=\frac{(-1)^{n-1} \pi}{(n-1) !} \cdot \frac{\partial^{n-1}}{\partial a^{n-1}} \cot (\pi a), \quad(n \geq 2) .
$$

From (20) and (21), we obtain that for a positive integer $n \geq 2$, if $\chi(-1)=(-1)^{n}$, then

$$
L(n, \chi)=\left.\frac{(-1)^{n-1} \pi}{2 q^{n} \cdot(n-1) !} \sum_{l=1}^{q} \chi(l) \frac{\partial^{n-1}}{\partial a^{n-1}} \cot (\pi a)\right|_{a=(1 / q)} .
$$

Applying Lemma 2 to the right side of (22), we claim that for a positive integer $n \geq 2$, if $\chi(-1)=(-1)^{n}$, then

$$
L(n, \chi)=i^{n-2} \frac{(-1)^{n-1} \pi^{n} \cdot 2^{n-1}}{q^{n} \cdot(n-1) !} \sum_{l=1}^{q} \chi(l) F\left(\frac{l}{q}, 1-n\right) .
$$

We know from Theorem 12.13 in [1] that for a nonnegative integer $n$,

$$
\zeta(-n, x)=-\frac{B_{n+1}(x)}{n+1}
$$

which implies

$$
\begin{aligned}
F\left(\frac{l}{q}, 1-n\right) & =\sum_{j=0}^{\infty} \sum_{r=1}^{q} \frac{e^{(2 \pi i(q j+r) l / q)}}{(q j+r)^{1-n}} \\
& =-\frac{1}{n q^{1-n}} \sum_{r=1}^{q} e^{(2 \pi i r l / q)} B_{n}\left(\frac{r}{q}\right) .
\end{aligned}
$$

Hence, by inserting (25) into (23), in view of (1), we get that for a positive integer $n \geq 2$, if $\chi(-1)=(-1)^{n}$, then

$$
L(n, \chi)=i^{n-2} \frac{(-1)^{n} \pi^{n} \cdot 2^{n-1}}{q \cdot n !} \sum_{r=1}^{q} B_{n}\left(\frac{r}{q}\right) G(r, \chi) .
$$

It follows from (26) that (15) and (16) hold true for a positive integer $n$. We next prove that the case $n=0$ in (16) is complete. In fact, since $B_{1}(x)=x-(1 / 2)$ (see, e.g., p. 266 in [1]), by the familiar geometric sum stated in Theorem 8.1 in [1] and the property of character sums described in Theorem 6.10 in [1], we discover that for an odd Dirichlet character $\chi$ modulo $q$,

$$
\begin{aligned}
\sum_{r=1}^{q} B_{1}\left(\frac{r}{q}\right) G(r, \chi) & =\sum_{l=1}^{q-1} \chi(l) \sum_{r=1}^{q}\left(\frac{r}{q}-\frac{1}{2}\right) e^{(2 \pi i r l / q)} \\
& =\frac{1}{q} \sum_{l=1}^{q-1} \chi(l) \sum_{r=1}^{q-1} r e^{(2 \pi i r l / q)} \\
& =\sum_{l=1}^{q-1} \chi(l) \frac{1}{e^{(2 \pi i l / q)}-1} \\
& =\frac{1}{2} \sum_{l=1}^{q-1} \chi(l)\left(\frac{e^{(\pi i l / q)}+e^{-(\pi i l / q)}}{e^{(\pi i l / q)}-e^{-(\pi i l / q)}}-1\right) \\
& =\frac{1}{2 i} \sum_{l=1}^{q-1} \chi(l) \cot \left(\frac{\pi l}{q}\right) .
\end{aligned}
$$

On the other hand, by taking $n=1$ in Lemma 1, in light of $s(n, n)=1$ for a nonnegative integer $n$ (see, e.g., p. 214 in $[17])$, we obtain that for an odd Dirichlet character $\chi$ modulo $q$, 


$$
\sum_{r=1}^{q-1} \chi(r) \cot \left(\frac{\pi r}{q}\right)=\frac{2 q}{\pi} L(1, \chi)
$$

Thus, combing (27) and (28) gives that for an odd Dirichlet character $\chi$ modulo $q$,

$$
\sum_{r=1}^{q} B_{1}\left(\frac{r}{q}\right) G(r, \chi)=\frac{q}{\pi i} L(1, \chi),
$$

which means (16) holds true for the case $n=0$. This completes the proof of Lemma 3.

Remark 2. Since the Bernoulli polynomials can be expressed by the Bernoulli numbers in the following way (see, e.g., Theorem 12.12 in [1]),

$$
B_{n}(x)=\sum_{k=0}^{n}\left(\begin{array}{l}
n \\
k
\end{array}\right) B_{k} x^{n-k}, \quad(n \geq 0),
$$

where $B_{k}$ is the $k$-th Bernoulli number; by applying (30) to Lemma 3 , one can easily get that if $n$ is a positive integer and $r \in\{0,1\}$ such that $r$ and $n$ have the same parity, and if $\chi(-1)=(-1)^{r}$,

$$
L(n, \chi)=(-1)^{r+1} i^{n} \frac{\pi^{n} \cdot 2^{n-1}}{q \cdot n !} \sum_{k=0}^{2\lfloor(n / 2)\rfloor}\left(\begin{array}{l}
n \\
k
\end{array}\right) B_{k} S(n-k, \chi),
$$

where $S(m, \chi)$ is given for a nonnegative integer $m$ by

$$
S(m, \chi)=\sum_{r=1}^{q}\left(\frac{r}{q}\right)^{m} G(r, \chi) .
$$

Formula (31) was firstly discovered by Alkan Theorem 1 in [18] using the Fourier expansions of the Bernoulli periodic functions and some properties of character sums and is also a key ingredient for the proofs of formulas (6) and (7). We here refer to Theorem 2 in [19] for an extension of (31). For a different proof of Lemma 3, one can consult to Theorem 1.3 in [20], where the author used the function equation for the Hurwitz zeta function to give the proof.

\section{The Proof of Theorem 1}

We are now in a position to provide the detailed proof of Theorem 1. It is well known that the Bernoulli polynomials satisfy the following difference equation (see, e.g., Theorem 12.14 in [1]):

$$
B_{n}(x+1)-B_{n}(x)=n x^{n-1}, \quad(n \geq 1),
$$

and the following addition formula (see, e.g., p. 275 in [1]):

$$
B_{n}(x+y)=\sum_{k=0}^{n}\left(\begin{array}{l}
n \\
k
\end{array}\right) B_{k}(x) y^{n-k}, \quad(n \geq 0) .
$$

Hence, we obtain from (33) and (34) that for a positive integer $m$,

$$
\begin{aligned}
x^{m} & =\frac{B_{m+1}(x+1)-B_{m+1}(x)}{m+1} \\
& =\frac{1}{m+1}\left\{\sum_{j=0}^{m+1}\left(\begin{array}{c}
m+1 \\
j
\end{array}\right) B_{j}(x)-B_{m+1}(x)\right\} \\
& =\frac{1}{m+1} \sum_{j=0}^{m}\left(\begin{array}{c}
m+1 \\
j
\end{array}\right) B_{j}(x) .
\end{aligned}
$$

It follows from (1), (35), and $B_{0}(x)=1$ (see, e.g., p. 266 [1]) that

$$
\begin{aligned}
\sum_{r=1}^{q}\left(\frac{r}{q}\right)^{m} G(r, \chi)= & \frac{1}{m+1} \sum_{j=0}^{m}\left(\begin{array}{c}
m+1 \\
j
\end{array}\right) \sum_{r=1}^{q} B_{j}\left(\frac{r}{q}\right) \sum_{l=1}^{q} \chi(l) e^{(2 \pi i l r / q)} \\
= & \frac{1}{m+1} \sum_{j=1}^{m}\left(\begin{array}{c}
m+1 \\
j
\end{array}\right) \sum_{r=1}^{q} B_{j}\left(\frac{r}{q}\right) \sum_{l=1}^{q} \chi(l) e^{(2 \pi i l r / q)} \\
& +\frac{1}{m+1} \sum_{l=1}^{q} \chi(l) \sum_{r=1}^{q} e^{(2 \pi i l r / q) .}
\end{aligned}
$$

Since $G( \pm q, \chi)=0$ for a nonprincipal Dirichlet character $\chi$ modulo $q$, if $\chi$ is a nonprincipal Dirichlet character modulo $q$, we have

$$
\begin{aligned}
\sum_{r=1}^{q} B_{j}\left(\frac{r}{q}\right) \sum_{l=1}^{q} \chi(l) e^{(2 \pi i l r / q)} & =\sum_{r=1}^{q-1} B_{j}\left(\frac{r}{q}\right) \sum_{l=1}^{q} \chi(l) e^{(2 \pi i l r / q)} \\
& =\sum_{r=1}^{q-1} B_{j}\left(1-\frac{r}{q}\right) \sum_{l=1}^{q} \chi(l) e^{(2 \pi i l(q-r) / q)} \\
& =(-1)^{j} \chi(-1) \sum_{r=1}^{q} B_{j}\left(\frac{r}{q}\right) \sum_{l=1}^{q} \chi(l) e^{(2 \pi i l r / q)},
\end{aligned}
$$

where we used the symmetric relation of the Bernoulli polynomials (see, e.g., p. 274 [1]) as follows:

$$
B_{n}(1-x)=(-1)^{n} B_{n}(x), \quad(n \geq 0) .
$$

Moreover, the familiar geometric sum implies that

$$
\sum_{l=1}^{q} \chi(l) \sum_{r=1}^{q} e^{(2 \pi i l r / q)}=0 .
$$

It follows from (1), (36), (37), and (39) that if $\chi$ is a nonprincipal even Dirichlet character modulo $q$, then

$$
\begin{aligned}
\sum_{r=1}^{q}\left(\frac{r}{q}\right)^{m} G(r, \chi) & =\frac{1}{m+1} \sum_{\substack{j=1 \\
2 \mid j}}^{m}\left(\begin{array}{c}
m+1 \\
j
\end{array}\right) \sum_{r=1}^{q} B_{j}\left(\frac{r}{q}\right) G(r, \chi) \\
& =\frac{1}{m+1} \sum_{j=1}^{\lfloor(m / 2)\rfloor}\left(\begin{array}{c}
m+1 \\
2 j
\end{array}\right) \sum_{r=1}^{q} B_{2 j}\left(\frac{r}{q}\right) G(r, \chi),
\end{aligned}
$$


and if $\chi$ is an odd Dirichlet character modulo $q$, then

$$
\begin{aligned}
\sum_{r=1}^{q}\left(\frac{r}{q}\right)^{m} G(r, \chi) & =\frac{1}{m+1} \sum_{\substack{j=1 \\
2 \nmid j}}^{m}\left(\begin{array}{c}
m+1 \\
j
\end{array}\right) \sum_{r=1}^{q} B_{j}\left(\frac{r}{q}\right) G(r, \chi) \\
& =\frac{1}{m+1} \sum_{j=0}^{\lfloor(m-1 / 2)\rfloor}\left(\begin{array}{c}
m+1 \\
2 j+1
\end{array}\right) \sum_{r=1}^{q} B_{2 j+1}\left(\frac{r}{q}\right) G(r, \chi) .
\end{aligned}
$$

Applying Lemma 3 to the right hand sides of (40) and (41), we get that if $\chi$ is a nonprincipal even Dirichlet character modulo $q$, then

$$
\sum_{r=1}^{q}\left(\frac{r}{q}\right)^{m} G(r, \chi)=-\frac{q}{m+1} \sum_{j=1}^{\lfloor(m / 2)\rfloor}\left(\begin{array}{c}
m+1 \\
2 j
\end{array}\right)(-1)^{j} \frac{(2 j) ! \cdot L(2 j, \chi)}{\pi^{2 j} \cdot 2^{2 j-1}},
$$

and if $\chi$ is an odd Dirichlet character modulo $q$, then

$$
\sum_{r=1}^{q}\left(\frac{r}{q}\right)^{m} G(r, \chi)=-\frac{q i}{m+1} \sum_{j=0}^{\lfloor(m-1 / 2)\rfloor}\left(\begin{array}{c}
m+1 \\
2 j+1
\end{array}\right)(-1)^{j} \frac{(2 j+1) ! \cdot L(2 j+1, \chi)}{\pi^{2 j+1} \cdot 2^{2 j}} .
$$

Now, (8) and (9) follow from (42) and (43), respectively. This concludes the proof of Theorem 1 .

\section{Data Availability}

No data were used to support the findings of the study.

\section{Conflicts of Interest}

The authors declare that they have no conflicts of interest.

\section{References}

[1] T. M. Apostol, Introduction to Analytic Number Theory, Springer, New York, NY, USA, 1976.

[2] B. C. Berndt and R. J. Evans, "The determination of Gauss sums," Bulletin of the American Mathematical Society, vol. 5, pp. 107-128, 1981.

[3] Z. Chen and W. Zhang, "On the fourth-order linear recurrence formula related to classical Gauss sums," Open Mathematics, vol. 15, pp. 1251-1255, 2017.

[4] S. Chowla, "On Gaussian sums," Proceedings of the National Academy of Sciences of the United States of America, vol. 48, pp. 1127-1128, 1962.

[5] J. Yang and W. Zheng, "On a theorem of Chowla," Journal of Number Theory, vol. 106, pp. 50-55, 2004.

[6] Y. Yi and W. P. Zhang, "On the $2 \mathrm{k}$-th power mean of inversion of L-functions with the weight of the Gauss sum," Acta Mathematica Sinica, English Series, vol. 20, no. 1, pp. 175-180, 2004.

[7] H. Liu and W. Zhang, "On the hybrid mean value of Gauss sums and generalized Bernoulli numbers," Proceedings of the Japan Academy, Series A, vol. 80, pp. 113-115, 2004.

[8] H. Liu and W. Zhang, "Hybrid mean value of generalized Bernoulli numbers, general Kloosterman sums and Gauss sums," Journal of the Korean Mathematical Society, vol. 44, pp. 11-24, 2007.
[9] E. Alkan, "On linear combinations of special values of L-functions," Manuscripta Mathematica, vol. 139, no. 3-4, pp. 473-494, 2012.

[10] Y. He, "Explicit expressions for finite trigonometric sums," Journal of Mathematical Analysis and Applications, vol. 484, p. 24, Article ID 123702, 2020.

[11] W. Zhang and X. Lin, "Identities involving trigonometric functions and Bernoulli numbers," Applied Mathematics and Computation, vol. 334, pp. 288-294, 2018.

[12] W. Zhang, Lecture Notes in Contemporary Mathematics, pp. 173-179, Science Press, Beijing, China, 1989.

[13] W. Zhang, "On the mean values of Dedekind sums," Journal de Théorie des Nombres de Bordeaux, vol. 8, pp. 429-442, 1996.

[14] N. E. Nörlund, Vorlesungen über Differenzenrechnung, Springer, Berlin, Germany, 1924.

[15] D. Cvijović and J. Klinowski, "Values of the Legendre chi and Hurwitz zeta functions at rational arguments," Mathematics of Computation, vol. 68, pp. 1623-1630, 1999.

[16] E. M. Ferreira, A. K. Kohara, and J. Sesma, "New properties of the Lerch's transcendent," Journal of Number Theory, vol. 172, pp. 21-31, 2017.

[17] L. Comtet, Advanced Combinatorics, the Art of Finite and Infinite Expansions, D. Reidel Publishing, Dordrecht, The Netherlands, 1974.

[18] E. Alkan, "Values of Dirichlet L-functions, Gauss sums and trigonometric sums," The Ramanujan Journal, vol. 26, no. 3, pp. 375-398, 2011.

[19] E. Alkan, "On Dirichlet L-functions with periodic coefficients and Eisenstein series," Monatshefte für Mathematik, vol. 163, no. 3, pp. 249-280, 2011.

[20] Y. He, "Several expressions of Dirichlet L-function at positive integers," Acta Mathematica Sinica, English Series, vol. 35, no. 9, pp. 1549-1562, 2019. 